\title{
Editorial: Cognitive Science and Artificial Intelligence for Human Cognition and Communication
}

\author{
Huimin $\mathrm{Lu}^{1} \cdot$ Yujie $\mathrm{Li}^{2}$ \\ Published online: 2 May 2019 \\ (C) Springer Science+Business Media, LLC, part of Springer Nature 2019
}

\section{Editorial}

Cognitive computing has broad horizons, which covers different characteristics of cognition. Moreover, cognitive science is interdisciplinary, scientific study of the human reasoning, emotions, language, perception, attention, and memory. However, artificial intelligence (AI) is to explore the design of computers and software that would capable of intelligent behavior. The integration of cognitive science and AI offers with a deepunderstanding of human cognition and communication. In addition, the creativeand technical skills are applying the knowledge in AI solutions and applications in engineering psychology. This special issue is forming connections between cognitive science and AI that examines human performance anddesign ofengineeringpsychology. Theearlierstudieshavebeen revealed powerful principles, methodologies, and algorithms, prompting in human self-examination and perception for building AI systems that match or better human performance. Thus, this special issue focusing on convergence of cognitive science and AI principles and methods would be helpful to improve the thinking ability skills of the humans in engineering psychology.

This special issue features six selected papers with high quality. The first article, "EEDVMI: Energy-Efficient Dynamic Virtual Machines Integration", authored by Yin Zhang, synthetically considered the influence of a multi-order Markov model and the CPU state at different times and proposed a novel Korder mixed Markov model for predicting the CPU load of the host for a period of time.

The second article titled "Deep-Sea Organisms Tracking Using Dehazing and Deep Learning" developed a method

Huimin Lu

dr.huimin.lu@ieee.org

Yujie Li

yzyjli@gmail.com

1 Kyushu Institute of Technology, 1-1 Sensuicho, Tobata Ward, Kitakyushu, Fukuoka 804-8550, Japan

2 Yangzhou University, Hanjiang, Yangzhou, Jiangsu, China

for underwater real-time recognition and tracking of multiobjects, which named as "You Only Look Once: YOLO". This method provided a very fast and accurate tracker.

In the next article with the title "Learning for Smart Edge: Cognitive Learning-Based Computation Offloading", the authors considered the problem of running the learning-based computation offloading scheme for the first time and propose the learning for smart edge architecture. They gave the computation offloading optimization problem of mobile devices under multi-user and multi edge cloud scenarios.

The fourth article titled "Human Emotion Recognition Using an EEG Cloud Computing Platform" proposed an emotional state evoked paradigm to identify the brain area where the emotion feature is most evident.

Aiming at improving the performance of the endmember extraction problem in hyperspectral images, a new extraction method based on discrete hybrid artificial bee colony algorithm and genetic algorithm (DABC_GA) is proposed in the fifth article, "Endmember Extraction of Hyperspectral Remote Sensing Images Based on an Improved Discrete Artificial Bee Colony Algorithm and Genetic Algorithm".

The last article titled "Detection of Circulating Tumor Cells in Fluorescence Microscopy Images Based on ANN Classifier" investigated an automatic CTCs identification method in fluorescence microscopy images.

Acknowledgements This work is partly supported by the Grant-In-Aid for Scientific Research from the Ministry of Education, Culture, Sports, Science and Technology of Japan under grant number (B26861131), Leading Initiative for Excellent Young Researcher of Ministry of Education, Culture, Sports, Science and Technology-Japan (16809746), Grants-in-Aid for Scientific Research of JSPS (17 K14694), Research Fund of The Telecommunications Advancement Foundation, and Fundamental Research Developing Association for Shipbuilding and Offshore, and Japan-China Scientific Cooperation Program (6171101454).

Publisher's Note Springer Nature remains neutral with regard to jurisdictional claims in published maps and institutional affiliations. 


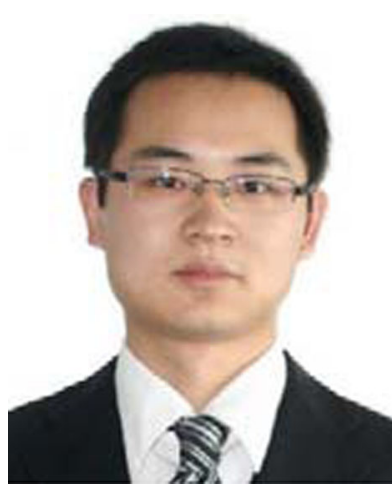

Huimin Lu received a B.S. degree in Electronics Information Science and Technology from Yangzhou University in 2008. $\mathrm{He}$ received M.S. degrees in Electrical Engineering from Kyushu Institute of Technology and Yangzhou University in 2011. He received a Ph.D. degree in Electrical Engineering from Kyushu Institute of Technology in 2014. From 2013 to 2016, he was a JSPS research fellow (DC2, $\mathrm{PD}$, and FPD) at Kyushu Institute of Technology. Currently, he is an Assistant Professor in Kyushu Institute of Technology and an Excellent Young Researcher of MEXT-Japan. His research interests include computer vision, robotics, artificial intelligence, and ocean observing.

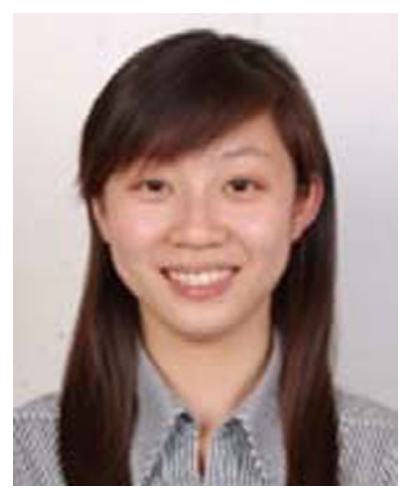

Yujie Li received the B.S. degree in Computer Science and Technology from Yangzhou University in 2009. She received M.S. degrees in Electrical Engineering from Kyushu Institute of Technology and Yangzhou University in 2012, respectively. She received a Ph.D. degree from Kyushu Institute of Technology in 2015. From 2016 to 2017, she was a Lecturer in Yangzhou University. Currently, she is an Assistant Professor in Fukuoka University, Japan and JSPS Research Fellow in Kyushu Institute of Technology, Japan. Her research interests include computer vision, sensors, and image segmentation. 